\title{
Process Modeling for the Fiber Diameter of Polymer, Spun by Pressure-Coupled Infusion Gyration
}

\author{
Xianze Hong, ${ }^{\dagger}$ Anthony Harker, ${ }^{\ddagger}$ and Mohan Edirisinghe ${ }^{* \dagger \dagger}$ \\ ${ }^{\dagger}$ Department of Mechanical Engineering, University College London (UCL), Torrington Place, London WC1E 7JE, U.K. \\ ${ }^{\ddagger}$ Department of Physics and Astronomy, University College London (UCL), Gower Street, London WC1E 6BT, U.K.
}

\section{Supporting Information}

ABSTRACT: Several new spinning methods have been developed recently to mass produce polymeric fibers. Pressure-coupled infusion gyration is one of them. Because the fiber diameter plays a pivotal role for the mechanical, electrical, and optical properties of the produced fiber mats, in this work, polyethylene oxide is used as a model polymer, and the processing parameters including polymer concentration, infusion (flow) rate, working pressure, and rotational speed are chosen as variables to control fiber diameters spanning the micro- to nanoscale. The experimental process is modeled using response surface methodology, both in linear and nonlinear fitting formats, to allow optimization of processing parameters. The successes of the fitted models are evaluated

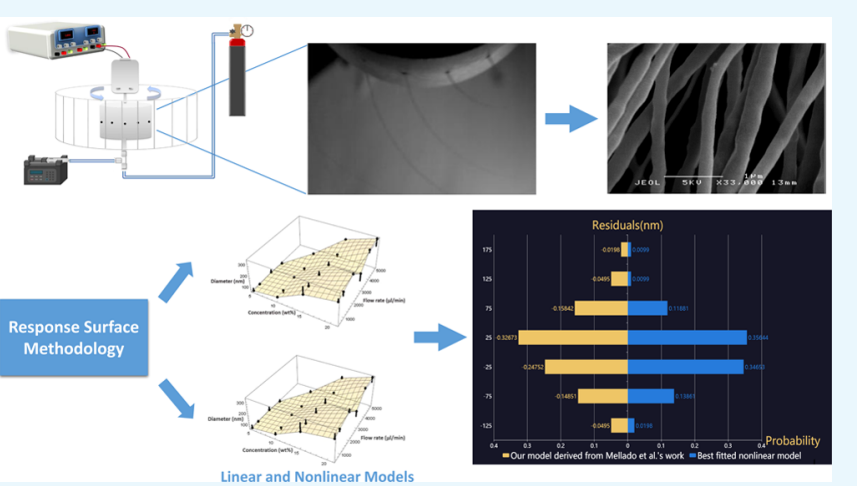
using adjusted $R^{2}$ and Akaike information criterion. A systematic description of the experimental process could be obtained according to the model in this study. From the analysis of variance, it is concluded that the polymer concentration of the solution and the working pressure affected the fiber diameters more strongly than other parameters.

\section{INTRODUCTION}

Polymeric fibers have a vast scope of applications and have recently garnered much attention owing to their various outstanding features such as high porosity, superior mechanical properties, and large surface area to volume ratio, which render them valuable in diverse applications such as cellular migration scaffolds, ${ }^{1-6}$ protective clothing, ${ }^{7-12}$ composite reinforcements, ${ }^{13,14}$ and nanosensors. ${ }^{15,16}$ Additionally, a well-aligned fibrous structure has potential for applications that require a particular orientation over nonwoven fibers (randomly interlaced), for example, the differentiation basement of tendon cells and peripheral nerves. ${ }^{17-20}$ Despite the versatility of nanofibers, their use is still restricted by the lack of proper quantity-production methods.

State of the art techniques used to fabricate fine fibers include phase separation, template synthesis, and electrospinning, with the latter being the most versatile and promising in the past decades; but the utilization of electrospinning is restricted by its many disadvantages such as random orientation of produced fibers, difficulty to build three-dimensional (3D) structures, sensitivity to solution electrical conductivity and jet stability, high cost and low safety due to applied high voltage ( $\mathrm{kV}$ level), and low production rate. Although its sister processes, for example, self-organized needleless (tube electrospinning) ${ }^{21,22}$ or the use of multiple needles, ${ }^{23}$ can improve the electrospinning productivity, they introduce side effects such as capillary effect (multiple needles) and increase the production cost by requiring a bigger size of equipment.
To overcome the major limitations of electrospinning, several methods have been explored that largely originate from the industrial jet-spinning technique. They make use of inexpensive centrifugation for low cost and large scale production of uniaxially aligned 3D fibrous bundles consistently, and they generate fibers with diameters ranging from micro- to nanoscale. These methods include centrifugal spinning, ${ }^{24-26}$ force spinning, ${ }^{27}$ pressurized gyration, ${ }^{28-32}$ infusion gyration, ${ }^{33}$ and pressure-coupled infusion gyration (PCIG). ${ }^{34}$ Many previous studies demonstrated that these techniques are capable of effectively producing a variety of products independent of the material properties. Experimental investigation into PCIG demonstrated that it can overcome discontinuous production of pressurized gyration and force spinning and is able to produce finer fibers than infusion gyration and centrifugal spinning, which are only really capable of generating fibers on submicron or micrometer level. ${ }^{34}$

PCIG is able to bring materials to the nanoscale not only to improve their properties and afford them new advanced features beyond bulk materials but also to produce uniaxially aligned 3D fibrous bundles consistently and cost-effectively. During rotational spinning, PCIG feeds the polymer solutions into the vessel continuously and utilizes gas blowing and highspeed rotation to extrude several parallel polymer jets from

Received: March 10, 2018

Accepted: May 2, 2018

Published: May 21, 2018 
orifices that are equally spaced on the surface of the vessel. Fibers with a controlled morphology can be prepared by selectively adjusting the processing parameters, for example, solution concentration, applied pressure, rotational speed, infusion (flow) rate and also, if necessary, collection distance. Compared with other gyration methods, the fiber produced using the PCIG method is much thinner. This is because the mass transfer of dynamic fluid flow can be regulated by the infusion rate of the solution when flowing across the orifice during spinning, and hence the input material can be effectively used to obtain the desired product morphology by balancing different combinations of processing parameters with solvent evaporation.

For some specific applications that are sensitive to the porosity and surface area (dominated by fiber diameter), for instance, cellular scaffolds, the fiber should be produced at a given diameter. However, PCIG is affected by various interacting parameters simultaneously; thus, to design an appropriate experimental process is complex for achieving a desired diameter, and the use of numerous trials to prepare fibers with the desired diameters would be expensive and timeconsuming. Researchers have tried to figure out scientific and forecasting tools in a variety of processes. Nowadays, there are several data mining and machine learning algorithms that can capably and reliably optimize the experimental process by quantifying the relationships between data using effective techniques such as Box-Behnken design, response surface methodology (RSM), and artificial neural networks (ANN), with the last one more popular now.

ANN and RSM are two widely used methods of mathematical modeling and computer simulation for computational processes. ${ }^{35,36}$ ANN consists of input, hidden, and output layers and is inspired by the working principles/structures of biological neural networks of the human brain. There are different populations of interconnected processing units (neurons) within layers, and these units are interconnected with each other to investigate their inter-relationships and predict the specific outputs that relate to different input data and specific functions used. ANN, more precisely, the three layers back propagation (BP) ANN has been used as a tool for modeling the electrospinning processes as well as for predicting the geometry and mechanical properties of the spun fibers. The units in the hidden layer can be manipulated as required; hence, any continuous functions can be represented with any precision. In addition, $\mathrm{BP}$ ANN is able to solve multivariate regression problems (linear/nonlinear) by training models through computing functions' gradient, then adjusting the weights and thresholds between neurons in each layer along the negative gradient direction, and to optimize the functions (also known as the gradient descent algorithm). ${ }^{37,38}$

However, despite the aforementioned strengths of ANN, there are also several known limitations of ANN, including: the slow convergence when the estimated value is close to the target; sensitivity to the noise of the training sample, that is, relatively poor training effect if there are intense changes in the sample; more notably, a tricky issue is that there is currently no general formula for the determination of the number of units (neurons) in the hidden layer, when the network structure is designed. As the number of the units directly determines the ability of the network to dig linear/nonlinear information from data, insufficient quantity of selected units may lead information to be expressed inadequately; an excessive quantity of units will increase the computational overhead exponentially, also resulting in a decline in network generalization. ${ }^{35-38}$

RSM is a convenient method that needs fewer experiments than ANN to construct an effective model. It shows good performance if the data are well-distributed statistically in the design of the experiment. ${ }^{39}$ On the basis of the training data, RSM uses statistical methods, taking into account the combined effects of several parameters to estimate the relationships (e.g., functional relevance) and the response of the modeling process by fitting an empirical model (linear/nonlinear). The resulting models can be evaluated and further used as a tool for predicting and optimizing the settings of the independent variables to reduce residuals. ${ }^{39,40}$ According to its structural characteristics, in respect of obtaining results of the interaction between different components for the whole system, sensitivity analysis, and other in-depth and advanced information, RSM has shown better performance in comparison with ANN. In some previous studies, RSM has been used for optimizing processes and material properties in different fields, for instance poly(vinyl alcohol) hydrogel, ${ }^{41}$ thermoplastic elastomers, ${ }^{42}$ and diamond-like carbon films. ${ }^{43}$

To have an overview of the effect of the PCIG experimental parameters individually and the quantitative basis of the relationships between PCIG parameters and the spun fiber diameter, RSM is used in this work. Using a mathematical model to describe the effects of PCIG parameters allows us to represent the influencing parameters in a simple and systematic way and to predict the response of the experiments with different parameter combinations. In addition, owing to the benefit of RSM, the number of experiments for testing the influences of all possible combinations of parameters on diameter could be reduced.

\section{MATERIALS AND METHODS}

2.1. Materials and Experiment Preparation. Poly(ethylene oxide) (PEO, molecular weight $\left(M_{\mathrm{w}}\right)$ of $2 \times 10^{5} \mathrm{~g}$ $\mathrm{mol}^{-1}$ ) purchased from Sigma-Aldrich (Poole, UK) is used asreceived, and deionized water is selected as the solvent. The PCIG apparatus used in this work (Figure 1a) consists of a cylindrical rotatable vessel $40 \mathrm{~mm}$ in height and $60 \mathrm{~mm}$ in diameter. It is equipped with 20 orifices symmetrically distributed on the vessel surface, each $0.5 \mathrm{~mm}$ in diameter. A $\mathrm{dc}$ motor joint is at the top of the vessel, and it can work in the range of $4.5-15 \mathrm{~V}$ to drive vessel rotation at various speeds up to $36000 \mathrm{rpm}$. The injected polymer solution flow is controlled by a syringe pump (PHD Ultra 4400, Harvard Apparatus Ltd., Edenbridge, UK) with a $10 \mathrm{~mL}$ syringe and connected at the bottom of the vessel by a plastic tube. A nitrogen gas cylinder is connected to the vessel bottom using a $\mathrm{T}$-junction to provide pressure (up to $0.3 \mathrm{MPa}$ ). The collector, a stationary copper mesh, is placed around the vessel at a preset distance of 100 $\mathrm{mm}$ to collect the jetted fibers. The distance between the orifices in the gyrator is $9 \mathrm{~mm}$, and the vertical copper wires in the collector are $25 \mathrm{~mm}$ apart. The spun fibers are assessed by means of a JSM-6301F scanning electron microscope, and the fiber diameters are determined by Image J software from random fibers at different locations per sample. A typical scanning electron microscopy (SEM) image of a spun fiber using PCIG is shown in Figure $1 \mathrm{~b}$.

2.2. Design of Experiments. To obtain a more systematic understanding of the processing conditions and to establish a quantitative basis for the relationships between PCIG parameters and the fiber diameter, RSM is employed. During 


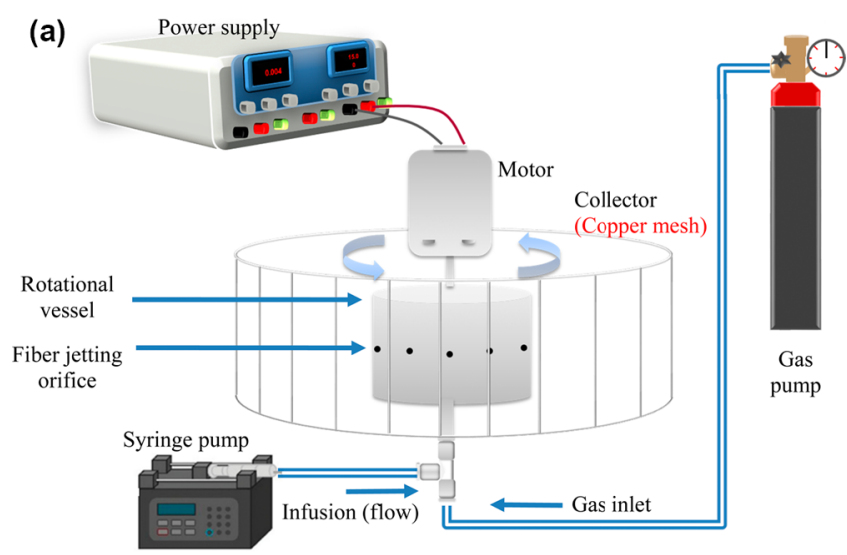

(b)

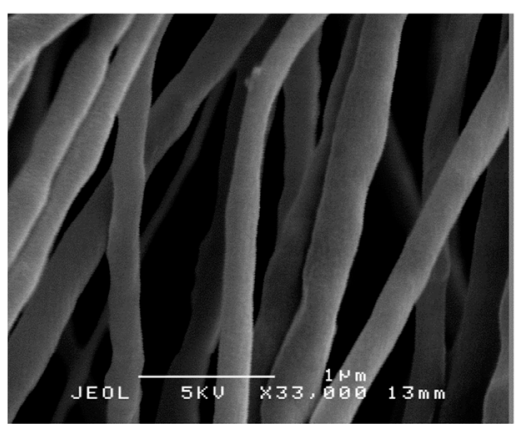

Figure 1. (a) PCIG fiber formation. (b) Typical SEM image of spun nanofiber using PCIG.

this study, fiber diameters are prepared by varying four parameters: PEO solution concentration, pressure, infusion (flow) rate, and rotation speed, and their relation to the fiber diameter is investigated using RSM. All other parameters including humidity $(38.5 \%)$ and ambient temperature $\left(25^{\circ} \mathrm{C}\right)$ are fixed during the experiments.

On the basis of the previous work, ${ }^{34}$ it is found that the appropriate distance of collection (from orifices to collector) is determined by the duration of solvent evaporation and the diameter of the spiral trajectory required for the polymer jets. An insufficient collection distance will result in the formation of thicker or coarser fibers, which are without enough elongation before reaching the collector. On the other hand, if the selected collection distance is beyond the critical value needed for evaporation of the solvent, the effect of further increase in the distance can be negligible. In this work, the orifice-collector distance is fixed at $100 \mathrm{~mm}$.

The infusion rate affects the hydrostatic pressure existing at the jetting orifices, and the volume and mass of polymer solution passing the orifice are also controlled by the infusion rate. A higher infusion rate will give a shorter solvent evaporation/polymer jet stretching time, hence promoting thicker and multiple polymer jets formed during spinning, which were observed in our previous work. ${ }^{34}$ Six levels of flow rate were chosen $(500,1000,2000,3000,4000$, and $5000 \mu \mathrm{L} /$ $\min )$ in this work.

The polymer concentration is one of the key factors in the spinning process; it selectively affects the formation and morphology of the spun fiber via adjusting solution's fluid properties, for example, the viscosity and chain entanglement degree, which is a prerequisite for uniform fiber formation. A higher polymer concentration helps to stabilize the polymer jets in the fiber generation process, which is crucial for the formation of a thicker fiber. In this work 5, 10, 15, and 21 wt \% are used as the four levels of solution concentration.

The influence of applied pressure is studied by performing experiments at three levels of pressure $(0.1-0.3 \mathrm{MPa})$. A high pressure would lead to a reduction in the fiber diameter at any fixed polymer concentration; this is attributed to the enhanced thinning effect introduced by gas blowing, and hence solvent evaporation that takes place in the radial direction of jets would also be facilitated by a higher applied pressure. In addition, the enhanced gas blowing would help to improve the instability of polymer jets at the orifices, which would promote the formation of the beaded or "defective" fiber.

The combination of solution blowing and centrifugal force act against the solution's surface tension and thus result in the deformation of the polymer droplets, thereby enabling the formation of fibers from the vessel orifice. The higher rotational speed will enhance this deformation by increasing the centrifugal force. The presence of non-Newtonian fluid shear stress acts against the normal stress and leads to tension and deformation along the same direction as the planar effect. ${ }^{28}$ Thus, the stretching in polymer jets will be further accelerated by the centrifugal force and gas blowing. The rotational speeds selected for this study are 10000,24000 , and $36000 \mathrm{rpm}$.

2.3. RSM Implementation. To capture the effects of all control parameters, 120 experiments were carried out for different combinations of parameters set at different levels. To achieve a high fitting degree, the input data should be measured accurately and should be sensitive to the parameters of the experimental process. For each individual experiment, over 100 fiber diameters were obtained from the random fibers of each sample, and each average diameter value was used as 1 input data to fit the model. However, there are 19 combinations, which give us droplets or beaded fibers, so the data of the remaining 101 experiments were used.

After measuring the fiber diameter related to each experimental data point, for describing the behavior of the response, it is necessary to fit a mathematical function based on the level of the study. For achieving a significant regression, the major part of variation of the experiment data must be wellfitted (explained) by the regression equation, and the remainder will certainly be induced by fitting residuals. Most of the variation of the residuals is caused by error, in this case, it is related to the spread of fiber diameters and random fluctuation during the fiber diameter measurement. We expect the spread of diameters to be the dominant effect. We used Mathematica to process the results of the experiments using the least-squares algorithm, which is a multiple regression analysis that is used to fit a mathematical model to a set of experimental data with the lowest residual. For that reason, both linear and nonlinear models were applied, as we assume linear or nonlinear mapping might exist between the parameters and fiber diameters. The goodness of the models that fit experimental data were compared using adjusted $R^{2}$ and the Akaike information criterion (AIC) test. The adjusted $R^{2}$ represents the level of the total variability that can be expected from the resulting model, adjusted by $R^{2}$ as more fitting variables were used. The AIC, a model selection criterion established on the basis of information entropy, can penalize the too complicated model (with redundant variables) by increasing its AIC value. A lower AIC value is preferred, as then smaller residuals exist between the fitting curve and the experimental data. Additionally, application of analysis of variance (ANOVA) was used to evaluate the quality of the 


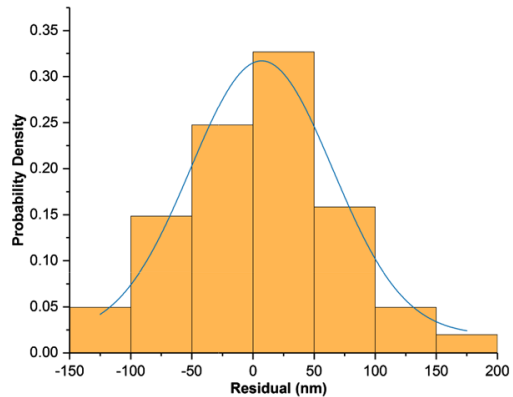

(a)

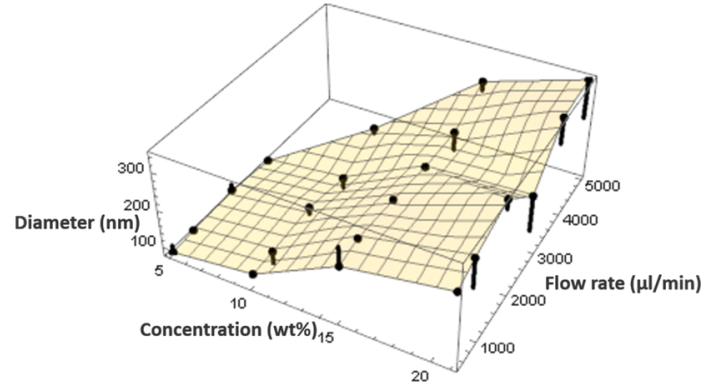

(b)

Figure 2. Residual distribution (a) and typical residual plot (b) of the power law model derived from Mellado et al.'s work ${ }^{25}$ (changing the infusion rate and polymer concentration, with a fixed pressure of $0.2 \mathrm{MPa}$ and a rotational speed of $36000 \mathrm{rpm}$ ).

fits, as the mathematical model found after fitting the function to the data sometimes does not satisfactorily describe the experimental domain under study of the linear models. The central idea of ANOVA was to compare the variations caused by changes of the levels of parameters with the variations by the random errors inherent in the measurements of the generated responses. Hence, it allows us to assess the source of the experimental variance and the significance of the regression used to predict the responses.

\section{RESULTS AND DISCUSSION}

We aim to find an optimal model to describe the relationship between the fiber diameter and processing parameters of PCIG [polymer concentration (c), infusion (flow) rate (f), pressure (p), and rotational speed (g)]; RSM is employed in this study. Introducing physical frameworks in the model building may help us have an in-depth understanding of the experimental process and the mechanism of fiber formation process and improve the goodness of the fitted models. Mellado et al. ${ }^{25}$ studied a basic theoretical rotary spinning framework to characterize the regimes of poly (lactic acid) nanofiber production. Their model can be expressed as

$$
r^{1.09 \pm 0.05} \approx \frac{a U^{1 / 2} v^{1 / 2}}{R_{\mathrm{c}}^{3 / 2} \Omega}
$$

where $r$ is the radius of the spun fiber; $v$ is the kinematic viscosity (calculated by extensional viscosity/density of solution); $R_{\mathrm{c}}$ is the collection distance that span a range from 90 to $180 \mathrm{~mm} ; \Omega$ is the rotational speed (varies from 4000 to $37000 \mathrm{rpm}) ; U$ is the mass velocity; and $a$ is the initial jet radius.

PCIG parameters affect the mean fiber diameter to different extents. The effects of each processing parameter on the fiber diameter are correlated and are presented in Figure $1 S$ of the Supporting information, which indicates that the polymer concentration (viscosity) and the mass velocity are positively correlated with the fiber diameter. Equation 1 also offers us some guidance, and the minimum rotational speed $\left(\Omega_{c}\right)$ for fiber formation is represented in eq 2

$$
\Omega_{\mathrm{c}} \approx \frac{\rho R_{\mathrm{c}}{ }^{2} \sigma^{2}}{a^{2}} \mu^{-3}
$$

It indicates that the rotational speed can be considered to have a power law relationship with other parameters, where $\mu$ is the extensional viscosity, $\rho$ is the density of the solution, and $\sigma$ is the surface tension. However, Mellado et al. ${ }^{25}$ did not incorporate additional pressure and flow rate parameters in their experiments. So, some mathematical expressions are suggested, which take into account the trends of variation of the fiber diameter displayed in Figure 1S, including a quartic relationship between the infusion (flow) rate and diameter, linear dependences are suggested with respect to concentration and pressure, and include a power. The fitting model is given in eq 3, which offers an AIC value of 1142.35 and adjusted $R^{2}$ of $95.1 \%$, and the coefficients are shown in Table 1S. The distribution of fitted residuals and a typical example of a residual plot (Figure 2; details of distribution and all other plots are shown in Table $2 S$ and Figure $2 S$ ) indicate that the residuals of the data point related with the current model vary dramatically. Hence, this is inadequate to make precise inferences about the data behavior in the experimental range of this work. It is noteworthy that the component of pressure in eq 3 has an extremely large power but is multiplied by a very small constant (i.e., $\beta_{7} \ll \beta_{8}$, as seen in Table $1 S$ ). It can be rewritten in this limit in the form of the exponential function for the future fittings, that is, $\mathrm{e}^{\beta \mathrm{p}}$.

$$
\begin{aligned}
y= & \beta_{0}\left(1+\beta_{1} f+\beta_{2} f^{2}+\beta_{3} f^{3}+\beta_{4} f^{4}\right)\left(1+\beta_{5} c\right)^{\beta_{6}} \\
& \left(1+\beta_{7} p\right)^{\beta_{8}} g^{\beta_{9}}
\end{aligned}
$$

Because PCIG is more complicated than the given framework of basic rotary spinning and the interactive effects between different parameters are not clearly known, for example, how the pressure affects solution flow or how the concentration affects viscosity through evaporation, it is essential to develop an empirical model based on the experimental data to allow establishment of a quantitative relationship between the fiber diameter and combined parameters.

3.1. Linear Model Fitting. The general equations for response $y$ (diameter) are developed by exploring fitting to multivariate experimental data using all possible polynomial functions of the variables up to a given order, which also can readily be extended to higher orders. Initially, we confine ourselves to linear combinations with variables up to second order forms, which offer a certain level of complexity to a model but without considering the trend of the experimental results, and its results can be used as another baseline (apart from the results of Mellado et al.'s ${ }^{25}$ work as presented in eq 3 ) to compare with each result of the following updates. The confidence interval we use for all fitting tests in this work is set to $95 \%$ by default. In the first fitting test, we look at functions that include all possible combinations of terms up to quadratic 
form in the processing parameters, which involves checking 16 384 nonrepeating combinations out of 1048 576. Additionally, effects of pressure and rotational speed act within an inverse relationship to the fiber diameter (as seen in Figure $1 \mathrm{Sc}, \mathrm{d}$ ), that is, increasing the pressure and speed will decrease the fiber diameter. So, it suggests that polynomials in the inverse of $p$ and $g(1 / p$ and $1 / g$ instead of $p$ and $g$ in the model fitting) might be better, which is also consistent with Mellado et al.'s ${ }^{25}$ work, as presented in eq 1 . The best-fitted quadratic model equation for the mean fiber diameter is given by eq 4 .

$$
\begin{aligned}
y= & \beta_{0}-\beta_{1} c+\beta_{2} c^{2}+\beta_{3} c f-\beta_{4} c / g-\beta_{5} /(g)^{2}-\beta_{6} / p \\
& +\beta_{7} c / p+\beta_{8} f / p+\beta_{9} /(g p)
\end{aligned}
$$

Here, $y$ is the mean fiber diameter $(y)$, and the value of each estimated coefficient $(\beta)$ can be found in Table 1 . It is worth

Table 1. ANOVA Table of the First Linear Model: Eq 4

\begin{tabular}{lllll} 
& $\begin{array}{c}\text { estimated coefficient } \\
\left(\beta_{n}\right)\end{array}$ & \multicolumn{1}{c}{ SS } & F-statistic & \multicolumn{1}{c}{$P$-value } \\
1 & 290.32 & & & \\
$c$ & -25.42 & $1.04 \times 10^{6}$ & 307.35 & $6.32 \times 10^{-31}$ \\
$c^{2}$ & 0.68 & 8020.60 & 2.37 & 0.13 \\
$c f$ & $9.63 \times 10^{-4}$ & $1.43 \times 10^{5}$ & 42.19 & $4.31 \times 10^{-9}$ \\
$c / g$ & $-1.09 \times 10^{5}$ & 2880.85 & 0.85 & 0.36 \\
$1 / g^{2}$ & $-6.84 \times 10^{10}$ & $3.49 \times 10^{4}$ & 10.30 & $1.83 \times 10^{-3}$ \\
$1 / p$ & -95.09 & $4.60 \times 10^{5}$ & 136.04 & $9.19 \times 10^{-20}$ \\
$c / p$ & 4.37 & $3.78 \times 10^{5}$ & 111.57 & $1.71 \times 10^{-17}$ \\
$f / p$ & $1.81 \times 10^{-3}$ & 7031.03 & 2.08 & 0.15 \\
$1 / g p$ & $2.32 \times 10^{6}$ & $7.70 \times 10^{4}$ & 22.74 & $7.00 \times 10^{-6}$ \\
\hline
\end{tabular}

noting that an overcomplicated expression may perform less well than the optimal expression; this is because the AIC and adjusted $R^{2}$ penalized the use of too many free parameters that actually do not contribute to improve (explain) the original variance. Figure $3 \mathrm{a}$ shows the distribution of the fitted residuals of the data points, and Figure $3 \mathrm{~b}$ is a typical plot that indicates how well eq 4 fits each data point. Its variance (ANOVA) table is shown in Table 1. SS is the sum of the squared observation deviations, and it can be dismembered in the sum of squares due to model residual and the sum of the square of regression. $P$-values represent the level of impact significances of the coefficients on the fiber diameter. As we use a 95\% confidence interval, if the $P$-value is larger than 0.05 , the related parameter has little impact on the mean fiber diameter based on the related fitting model, and it also reveals the quality of the current model. Table 1 indicates that the regressed coefficients of $c^{2}, c / g, f / p$ are quite random, and the obtained adjusted AIC and $R^{2}$ are 1118.93 and $86.2 \%$, which show that the quality of the fit is not very good, as to find the major explanatory variables, the adjusted $R^{2}$ should be $\geq 95 \%$.

To improve the model, visual inspection of the details of the experimental data and residual plots can help to generate valuable guides to the model suitability by denoting a response that indicates the kind of terms that need to be added to the model for improvement. Figure $1 \mathrm{Sb}$ suggests that there is a two-peaked dependence on the parameter of flow rate $(f)$; so going to the fourth power of $f$ will help to improve our model.

$$
\begin{aligned}
y= & \beta_{0}-\beta_{1} c+\beta_{2} c^{2}+\beta_{3} f-\beta_{4} f^{2}+\beta_{5} c f^{2}+\beta_{6} f^{3}-\beta_{7} f^{4} \\
& -\beta_{8} c / g-\beta_{9} /(g)^{2}-\beta_{10} / p+\beta_{11} c / p+\beta_{12} f / p \\
& +\beta_{13} /(g p)^{2}
\end{aligned}
$$

Equation 5 shows the linear model with the best AIC out of 524287 nonrepeating combinations of processing parameters that are needed to test, after the power of infusion rate is pushed up to fourth, which gives the AIC of 1107.22 and adjusted $R^{2}$ of $88.1 \%$. The ANOVA table, as shown in Table 2,

Table 2. ANOVA Table of the Linear Model with a Quartic Form of the Flow Rate, Inversed Pressure, and Rotational Speed Parameters: Eq 5

\begin{tabular}{lllll} 
& $\begin{array}{c}\text { estimated coefficient } \\
\left(\beta_{n}\right)\end{array}$ & \multicolumn{1}{c}{ SS } & F-statistic & \multicolumn{1}{c}{$P$-value } \\
1 & 137.99 & & & \\
$c$ & 23.45 & $1.04 \times 10^{6}$ & 357.17 & $1.51 \times 10^{-32}$ \\
$c^{2}$ & 0.63 & 8020.60 & 2.75 & 0.10 \\
$f$ & 0.40 & $1.21 \times 10^{5}$ & 41.66 & $5.94 \times 10^{-9}$ \\
$f^{2}$ & $2.86 \times 10^{-4}$ & $1.95 \times 10^{4}$ & 6.70 & 0.01 \\
$f^{2}$ & $2.92 \times 10^{-7}$ & $1.81 \times 10^{4}$ & 6.23 & 0.01 \\
$f^{3}$ & $7.17 \times 10^{-8}$ & $3.16 \times 10^{4}$ & 10.85 & $1 \times 10^{-3}$ \\
$f^{4}$ & $6.03 \times 10^{-12}$ & $1.76 \times 10^{4}$ & 6.04 & 0.02 \\
$c / g$ & $1.06 \times 10^{5}$ & 2550.71 & 0.88 & 0.35 \\
$g^{2}$ & $6.95 \times 10^{-10}$ & $3.35 \times 10^{4}$ & 11.51 & $1 \times 10^{-3}$ \\
$1 / p$ & 97.10 & $4.59 \times 10^{5}$ & 157.71 & $3.07 \times 10^{-21}$ \\
$c / p$ & 4.30 & $3.67 \times 10^{5}$ & 125.86 & $1.38 \times 10^{-18}$ \\
$f / p$ & $2.80 \times 10^{-3}$ & $1.04 \times 10^{4}$ & 3.56 & 0.06 \\
$g / p$ & $2.34 \times 10^{-6}$ & $7.67 \times 10^{4}$ & 26.33 & $1.73 \times 10^{-6}$
\end{tabular}

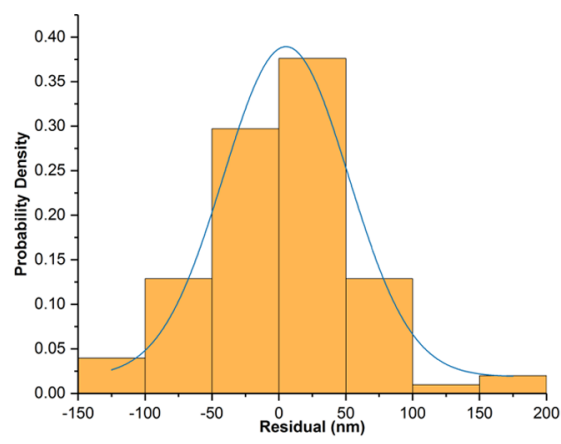

(a)

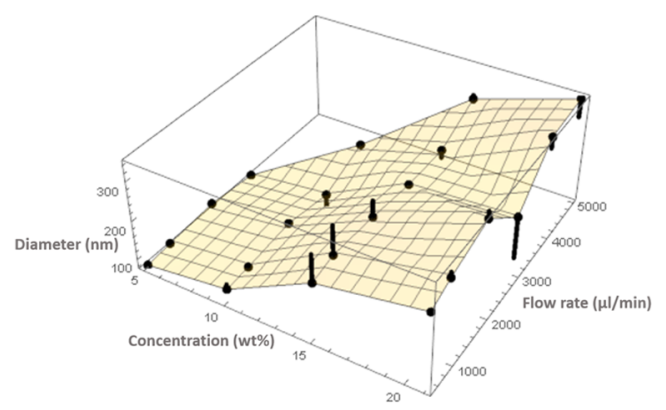

(b)

Figure 3. Residual distribution (a) and typical residual plot (b) of the first linear model (changing the infusion rate and polymer concentration, with a fixed pressure of $0.2 \mathrm{MPa}$ and a rotational speed of $36000 \mathrm{rpm}$ ). 


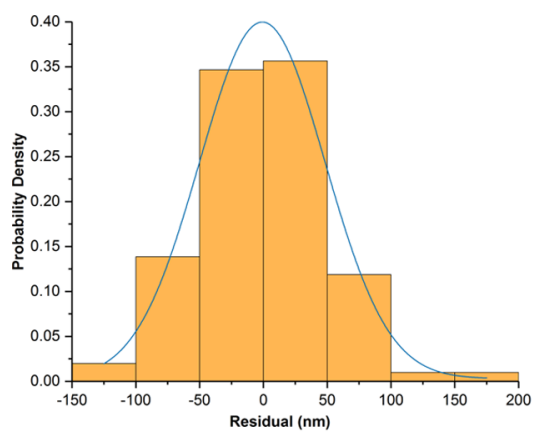

(a)

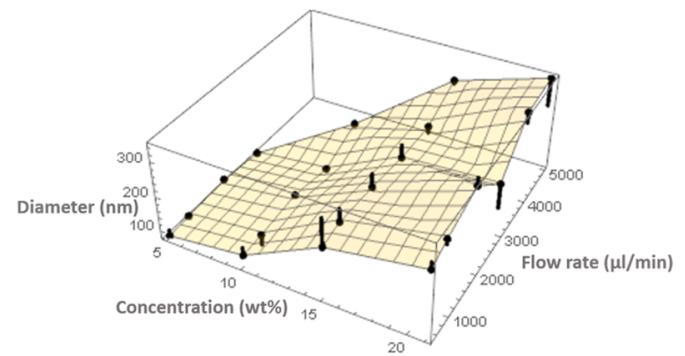

(b)

Figure 4. Residual distribution (a) and typical residual plot (b) of the fourth power incorporated linear model (changing the infusion flow rate and polymer concentration, with a fixed pressure of $0.2 \mathrm{MPa}$ and a rotational speed of $36000 \mathrm{rpm}$ ).

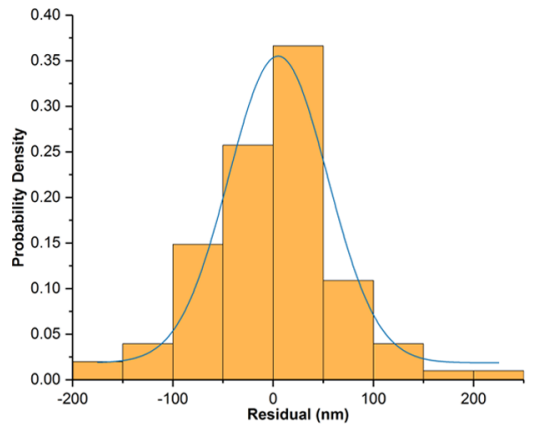

(a)

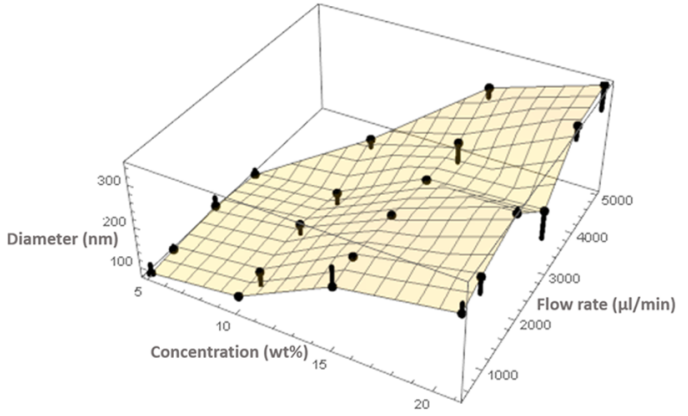

(b)

Figure 5. Residual distribution (a) and typical residual plot (b) of the first nonlinear model (changing the infusion rate and polymer concentration, with a fixed pressure of $0.2 \mathrm{MPa}$ and a rotational speed of $36000 \mathrm{rpm}$ ).

clearly indicates that the given estimated coefficient of the related model is more precise than the previous linear models, and the estimated coefficients are more significant than the previous two at a $95 \%$ confidence interval for the spun fibers. Also, the terms of concentration, pressure $^{-1}$, flow rate, and interactive terms between concentration and pressure ${ }^{-1}$ have shown a significant influence on the mean diameter of spun fibers. The addition of the fourth power terms in $f$ has certainly helped to improve the fitting performance. Figure 4 indicates that the data are better fitted and the residuals are effectively reduced.

The goodness of fitting of the linear test we applied does not give a high adjusted $R^{2}$ (up to $88.1 \%$ ), and we know that more parameters used in the function would help to improve the fitting precision. However, the number of fitting parameters in eq 5 has already reached 14 (but the observed fiber measurements give just 101 data). Continuously increasing the complexity of the fitting function may lead to overfitting because its excess estimated parameters would make the equation less generalized. Hence, it suggests that nonlinear methods could be used to update the model, as more complicated functions can be achieved using fewer parameters, and we are edging above the rule of thumb of no more than 1 fitting parameter per 10 measurements. Additionally, the estimate of error variance, also known as the estimation variance, is a benchmark showing the variance in fitting parameters. It is based on the sum of square error and takes into account the number of used parameters in the fitting and can be used to measure the goodness of fit.
3.2. Nonlinear Model Fitting. In contrast with the linear quadratic model, which has a general type of function that consists of simple multiplication and summation between parameters up to the second order, the forms of nonlinear model are almost infinite, including the incorporation of exponential functions, trigonometric functions, and so forth. To perform the trials on all possible combinations of the nonlinear component is computationally expensive. Hence, we inspect each graph in Figure $1 \mathrm{~S}$ based on our experience and combine all empirical components together to perform a nonlinear fitting. From Figure 1Sa, a similar quartic form applies to the dependence on $f$ under all conditions. We pick up the dependence on $f$ from the last best-fitted linear function, as it gives us an obvious improvement after we change the parameter of $f$ up to the fourth power, and the others are kept in linear forms. The fitted nonlinear function with the best AIC is given by eq 6 (the estimated coefficients as seen in Table 3S).

$$
\begin{aligned}
y= & \beta_{0}\left(1+\beta_{1} f-\beta_{2} f^{2}+\beta_{3} f^{3}-\beta_{4} f^{4}\right)\left(1+\beta_{5} c\right) \\
& \left(1+\beta_{6} / p\right)\left(1+\beta_{7} / g\right)
\end{aligned}
$$

The resulting adjusted $R^{2}$ is $94.9 \%$, and the lowest AIC that could be achieved is 1145.23 . The residual distribution and a typical residual plot are shown in Figure 5. The obtained estimate of variance is 4470.67 . This is much better in terms of adjusted $R^{2}$. However, a much higher AIC is given in this test. This is because the adjusted $R^{2}$ would be increased only if the new fitting parameter improves the model over the expected, and we only use eight coefficients in the current test, which is 


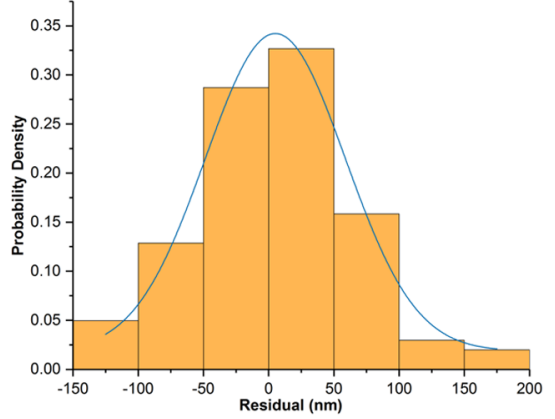

(a)

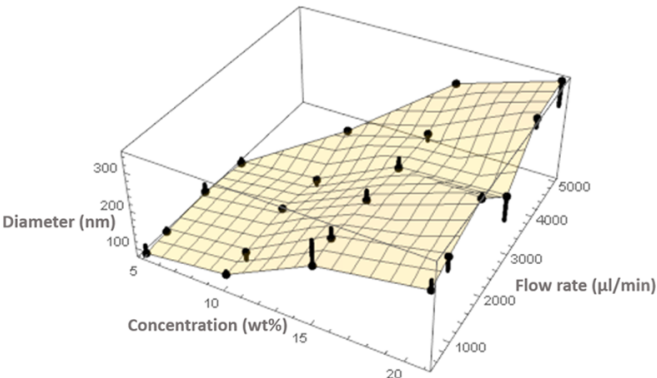

(b)

Figure 6. Residual distribution (a) and typical residual plot (b) of the nonlinear model with quadratic concentration and rotational speed terms but linear pressure terms (changing the infusion rate and polymer concentration, with a fixed pressure of $0.2 \mathrm{MPa}$ and a rotational speed of $36000 \mathrm{rpm}$ ).

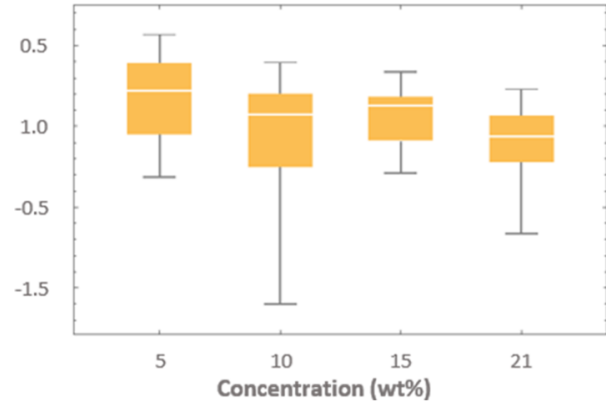

(a)

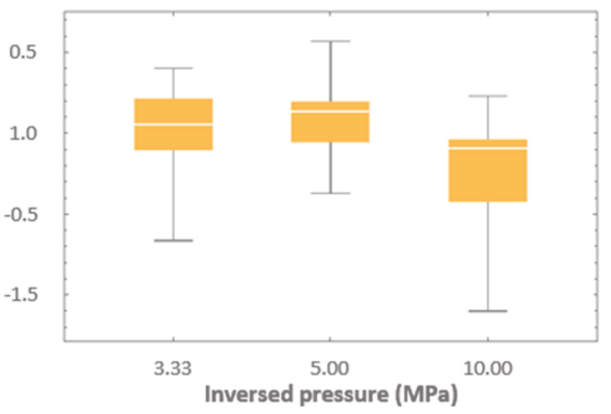

(c)

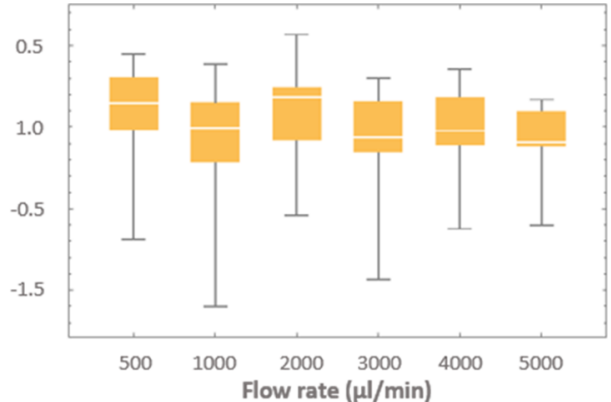

(b)

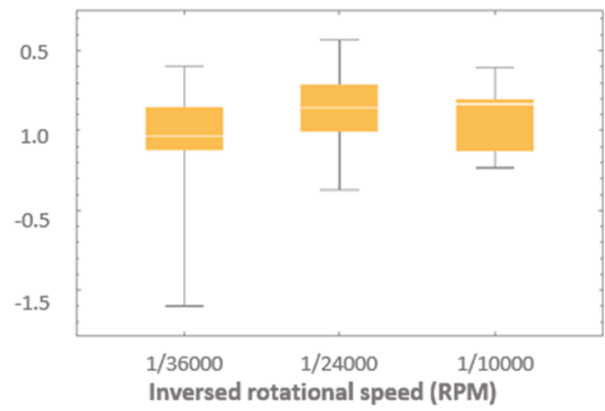

(d)

Figure 7. Box-Whisker charts of fractional residuals versus the experimental parameters: (a) concentration, (b) flow rate, (c) inverse pressure, and (d) inverse rotational speed.

less than those in any previous functional models in the linear fitting work; so this AIC value is perhaps acceptable. It is necessary to find suitable terms that can accurately describe the training data as well as the optimal compromises between the possible high adjusted $R^{2}$ and low AIC value, which need to be achieved.

According to the fitted result of the last linear model presented in eq 5 (with AIC of 1107.22 and the trends of the data variations, as shown in Figure $1 \mathrm{Sa}, \mathrm{c}, \mathrm{d})$, it is suggested that there are more appropriate quadratic terms for the parameters of solution concentration and rotational speed but linear dependence on pressure. Thus, we increase the order of $c$ and $g$ up to second. The optimized equation is given below (eq 7 and the estimated coefficients as listed in Table 4S)

$$
\begin{aligned}
y= & \beta_{0}\left(1+\beta_{1} f+\beta_{2} f^{2}+\beta_{3} f^{3}+\beta_{4} f^{4}\right)\left(1+\beta_{5} c+\beta_{6} c^{2}\right) \\
& \left(1+\beta_{7} / p\right)\left(1+\beta_{8} / g+\beta_{9} /(g)^{2}\right)
\end{aligned}
$$

The AIC is decreased to 1132.72, and the adjusted $R^{2}$ is $95.5 \%$. The quadratic form of subfunction dependence on the solution concentration and rotational speed and the linear terms of pressure can help to improve our fitting performance ( 3880.16 of the estimated variance) by offering a lower residual, as shown in Figure 6.

For improving the model one step further, we need to find what part of function space is giving the biggest deviation as well as the biggest residuals. The Box-Whisker charts in Figure 7 show the influences of fluctuation in the experimental parameters by using fractional residuals as a fraction of the mean diameters; they also indicate the median, 25 and $75 \%$ quartiles, as well as upper and lower bounds as a function of the concentration, flow rate, pressure, and rotational speed. It should be noted that the pressure and rotational speed are in their inverse forms.

Figure 7 indicates that the deviations of residuals are random, and some are very large. The lower rotational speed and higher 
concentration give the relatively lower deviations. It suggests that there may be systematic relationships, which exist in the pressure and rotational speed deviations. The random deviation is probably because the robust process of PCIG is a quantitatively tuned method for the fabrication of nanofibers but with a compromise of precise control of parameters that affects the mean fiber diameter.

The mean values of the experimental parameters chosen in the experiments are listed in Table 3, which indicates that the

Table 3. Mean of the Experimental Parameter Used in the PCIG Process

\begin{tabular}{lr} 
processing parameters & \multicolumn{1}{c}{ mean } \\
concentration $(\mathrm{wt} \%)$ & 13.86 \\
flow rate $(\mu \mathrm{L} / \mathrm{min})$ & 2311.88 \\
pressure $(\mathrm{MPa})$ & 0.20 \\
rotational speed $(\mathrm{rpm})$ & 29505.00 \\
diameter $(\mathrm{nm})$ & 251.91
\end{tabular}

constant terms of $f$ and $g$ in the model are possibly negligible because of the small coefficients with a very low multiplying power at the beginning of the functions. Presenting this change on graphs shows that the curves are forced to pass the origin. It is also sensible from the physical point of view of the PCIG process, as it explains as that if the infusion rate is decreased to be infinitely small, the resulting fibers will have an infinitely small diameter. Taking into account all optimizations we raised so far, the most up-to-date model can be presented as

$$
\begin{aligned}
y= & \left(\beta_{0} f+\beta_{1} f^{2}+\beta_{2} f^{3}+\beta_{3} f^{4}\right)\left(1+\beta_{4} c+\beta_{5} c^{2}\right) \\
& \mathrm{e}^{\beta_{6} / p}\left(1 / g+\beta_{7} /(g)^{2}\right)
\end{aligned}
$$

Figure 8 (all the other residual plots of this test are shown in Figure 3S) shows that the residuals have been reduced in the above model ( 3800.86 of estimated error variance), and the residual for each experimental parameter combination is listed in Table 5S. The obtained AIC value is 1128.83 , and the adjusted $R^{2}$ is $95.7 \%$. The relevant confidence intervals of the coefficients of pressure, concentration, and rotational speed are fairly standard, and the interval between 5 and $95 \%$ cumulative probabilities are listed in Table 4.

Ongoing work deals with the experimental verification of the model predictions, and early indications show that the model predictions are being satisfied.
Table 4. Confidence Interval of the Fitting Parameters in the Best-Fitted Nonlinear Model: Eq 8

\begin{tabular}{lll} 
& estimated coefficient $\left(\beta_{n}\right)$ & \multicolumn{1}{c}{ confidence interval } \\
$\beta_{0}$ & 1818.47 & $(-477.27,4114.22)$ \\
$\beta_{1}$ & -1.20 & $(-2.73,0.33)$ \\
$\beta_{2}$ & $3.04 \times 10^{-4}$ & $\left(-8.64 \times 10^{-5}, 6.95 \times 10^{-4}\right)$ \\
$\beta_{3}$ & $-2.56 \times 10^{-8}$ & $\left(-5.88 \times 10^{-8}, 7.60 \times 10^{-9}\right)$ \\
$\beta_{4}$ & 0.12 & $(-0.23,0.47)$ \\
$\beta_{5}$ & $9.26 \times 10^{-3}$ & $\left(3.38 \times 10^{-3}, 1.51 \times 10^{-2}\right)$ \\
$\beta_{6}$ & 0.14 & $(0.13,0.16)$ \\
$\beta_{7}$ & -7046.28 & $(-7455.68,-6636.89)$ \\
\hline
\end{tabular}

\section{CONCLUSIONS}

In this paper, RSM is applied to investigate the novel PCIG process for nanofiber making. ANOVA (variance) is carried out at the significance level of $5 \%$ to study the effects of the input parameters on the average fiber diameter, including infusion rate $(500-5000 \mu \mathrm{L} / \mathrm{min})$, rotational speed (10 000-36000 $\mathrm{rpm})$, applied pressure $(0.1-0.3 \mathrm{MPa})$, and polymer concentration (5-21 wt \%). To use this method in experimental optimization, it is necessary to select an appropriate experimental design to fit a mathematical formulation for evaluating the quality and accuracy of the fitting model based on the obtained experimental data. It is concluded that the best linear model gives an AIC of 1107.22 with a coefficient of determination (adjusted $R^{2}$ ) of $88.1 \%$ and indicates that the PEO polymer concentration and pressure had more significant effects than other parameters, for example, rotational speed on the spun fiber diameter. The best nonlinear model that we achieved offers an AIC of 1128.83 and a coefficient of determination of $95.7 \%$. Our model is useful for predicting and optimizing the process of fabrication of other types of nanofibers, using the PCIG method. However, it should be noted that the effects of physical properties of polymer solution, for example, viscosity and surface tension that have large differences based on different type of polymers, are not considered in this model. This could be addressed in the subsequent work.

\section{ASSOCIATED CONTENT}

\section{Supporting Information}

The Supporting Information is available free of charge on the ACS Publications website at DOI: 10.1021/acsomega.8b00452.

General trends of fiber diameter variation with different experimental parameters, the corresponding residual

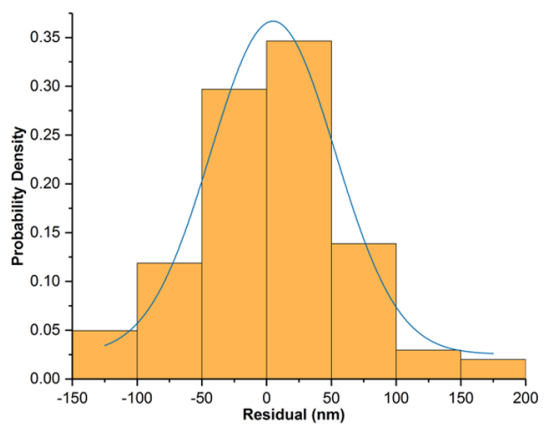

(a)

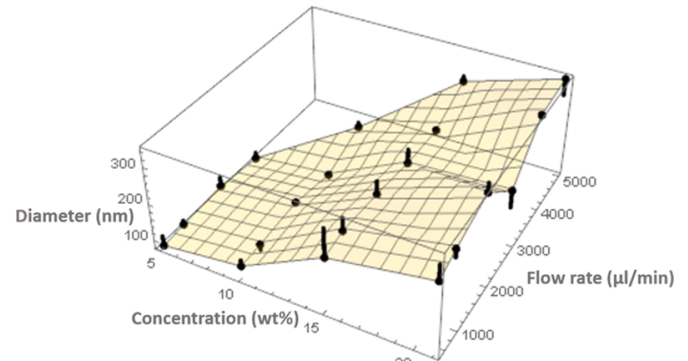

(b)

Figure 8. Residual distribution (a) and typical residual plot (b) of the optimized nonlinear model (changing the infusion rate and polymer concentration, with a fixed pressure of $0.2 \mathrm{MPa}$ and a rotational speed of $36000 \mathrm{rpm}$ ). 
plots of the nonlinear model derived from Mellado et al.'s work: eq 3 , the corresponding residual plots of the nonlinear model: eq 8, the estimated coefficients of the nonlinear model derived from Mellado et al.'s work: eq 3, the fitting residual of the nonlinear model derived from Mellado et al.'s work: eq 3, the estimated coefficients of the first nonlinear model: eq 6 , the estimated coefficients of the nonlinear model: eq 7 , and the fitting residual of the nonlinear model: eq 8 (PDF)

\section{AUTHOR INFORMATION}

\section{Corresponding Author}

*E-mail: m.edirisinghe@ucl.ac.uk (M.E.).

ORCID

Mohan Edirisinghe: 0000-0001-8258-7914

Notes

The authors declare no competing financial interest.

\section{ACKNOWLEDGMENTS}

The authors like to thank the Engineering and Physical Sciences Research Council (EPSRC), UK for providing the financial support for exploitation of pressurised gyration research (EP/L 023059/1 and EP/N 034228/1). Data supporting this study are provided in the paper and in the Supporting Information.

\section{REFERENCES}

(1) Xie, J.; Li, X.; Lipner, J.; Manning, C. N.; Schwartz, A. G.; Thomopoulos, S.; Xia, Y. "Aligned-to-random" nanofiber scaffolds for mimicking the structure of the tendon-to-bone insertion site. Nanoscale 2010, 2, 923-926.

(2) Xu, X.-Y.; Li, X.-T.; Peng, S.-W.; Xiao, J.-F.; Liu, C.; Fang, G.; Chen, K. C.; Chen, G.-Q. The behavior of neural stem cells on polyhydroxyalkanoate nanofiber scaffolds,. Biomaterials 2010, 31, 3967-3975.

(3) Liu, W.; Yeh, Y.-C.; Lipner, J.; Xie, J.; Sung, H.-W.; Thomopoulos, S.; Xia, Y. Enhancing the Stiffness of Electrospun Nanofiber Scaffolds with a Controlled Surface Coating and Mineralization. Langmuir 2011, 27, 9088-9093.

(4) Kumar, G.; Tison, C. K.; Chatterjee, K.; Pine, P. S.; McDaniel, J. H.; Salit, M. L.; Young, M. F.; Simon, C. G. The determination of stem cell fate by $3 \mathrm{D}$ scaffold structures through the control of cell shape. Biomaterials 2011, 32, 9188-9196.

(5) Pant, H. R; Bajgai, M. P.; Nam, K. T.; Seo, Y. A.; Pandeya, D. R.; Hong, S. T.; Kim, H. Y. Electrospun nylon-6 spider-net like nanofiber mat containing $\mathrm{TiO}_{2}$ nanoparticles: a multifunctional nanocomposite textile material. J. Hazard. Mater. 2011, 185, 124-130.

(6) Parhizkar, M.; Sofokleous, P.; Stride, E.; Edirisinghe, M. Novel preparation of controlled porosity particle/fiber loaded scaffolds using a hybrid micro-fluidic and electrohydrodynamic technique. Biofabrication 2014, 6, 045010.

(7) Yoon, B.; Lee, S. Designing Waterproof Breathable Materials Based on Electrospun Nanofibers and Assessing the Performance Characteristics. Fibers Polym. 2011, 12, 57-64.

(8) Faccini, M.; Vaquero, C.; Amantia, D. Development of Protective Clothing Against Nanoparticle Based on Electrospun Nanofibers. J. Nanomater. 2012, 2012, 892894.

(9) Zhang, H.; Wang, J.; Liu, X.; Wang, Z.; Wang, S. High Performance Self-Healing Epoxy/Polyamide Protective Coating Containing Epoxy Microcapsules and Polyaniline Nanofibers for Mild Carbon Steel. Ind. Eng. Chem. Res. 2013, 52, 10172-10180.

(10) Mahmoudian, M. R.; Alias, Y.; Basirun, W. J. Effect of narrow diameter polyaniline nanotubes and nanofibers in polyvinyl butyral coating on corrosion protective performance of mild steel. Prog. Org. Coat. 2012, 75, 301-308.
(11) Lee, M. W.; An, S.; Lee, C.; Liou, M.; Yarin, A. L.; Yoon, S. S. Self-healing transparent core-shell nanofiber coatings for anticorrosive protection. J. Mater. Chem. A 2014, 2, 7045-7053.

(12) Sehaqui, H.; Morimune, S.; Nishino, T.; Berglund, L. A. Stretchable and Strong Cellulose Nanopaper Structures Based on Polymer-Coated Nanofiber Networks: An Alternative to Nonwoven Porous Membranes from Electrospinning. Biomacromolecules 2012, 13, 3661-3667.

(13) Al-Saleh, M. H.; Sundararaj, U. Review of the mechanical properties of carbon nanofiber/polymer composites. Composites, Part A 2011, 42, 2126-2142.

(14) Dimchev, M.; Caeti, R.; Gupta, N. Effect of carbon nanofibers on tensile and compressive characteristics of hollow particle filled composites. Mater. Des. 2010, 31, 1332-1337.

(15) Liu, C.; Hayashi, K.; Toko, K. Au nanoparticles decorated polyaniline nanofiber sensor for detecting volatile sulfur compounds in expired breath. Sens. Actuators, B 2012, 161, 504-509.

(16) Zhang, H.; Li, Z.; Liu, L.; Xu, X.; Wang, Z.; Wang, W.; Zheng, W.; Dong, B.; Wang, C. Enhancement of hydrogen monitoring properties based on $\mathrm{Pd}-\mathrm{SnO}_{2}$ composite nanofibers. Sens. Actuators, $B$ 2010, 147, 111-115.

(17) Liu, W.; Thomopoulos, S.; Xia, Y. Electrospun Nanofibers for Regenerative Medicine. Adv. Healthcare Mater. 2012, 1, 10-25.

(18) Xie, J.; MacEwan, M. R.; Liu, W.; Jesuraj, N.; Li, X.; Hunter, D.; Xia, Y. Nerve Guidance Conduits Based on Double-Layered Scaffolds of Electrospun Nanofibers for Repairing the Peripheral Nervous System. ACS Appl. Mater. Interfaces 2014, 6, 9472-9480.

(19) Huang, C.; Ouyang, Y.; Niu, H.; He, N.; Ke, Q.; Jin, X.; Li, D.; Fang, J.; Liu, W.; Fan, C.; Lin, T. Nerve Guidance Conduits from Aligned Nanofibers: Improvement of Nerve Regeneration through Longitudinal Nanogrooves on a Fiber Surface. ACS Appl. Mater. Interfaces 2015, 7, 7189-7196.

(20) He, X.; Xiao, Q.; Lu, C.; Wang, Y.; Zhang, X.; Zhao, J.; Zhang, W.; Zhang, X.; Deng, Y. Uniaxially Aligned Electrospun All-Cellulose Nanocomposite Nanofibers Reinforced with Cellulose Nanocrystals: Scaffold for Tissue Engineering. Biomacromolecules 2014, 15, 618-627.

(21) Niu, H.; Lin, T. Fiber Generators in Needleless Electrospinning. J. Nanomater. 2012, 2012, 725950.

(22) Li, D.; Wu, T.; He, N.; Wang, J.; Chen, W.; He, L.; Huang, C.; EI-Hamshary, H. A.; Al-Deyab, S. S.; Ke, Q.; Mo, X. Threedimensional polycaprolactone scaffold via needleless electrospinning promotes cell proliferation and infiltration. Colloids Surf., B 2014, 121, $432-443$.

(23) Zhou, F.-L.; Gong, R.-H.; Porat, I. Mass production of nanofiber assemblies by electrostatic spinning. Polym. Int. 2009, 58, 331-342.

(24) Ren, L.; Pandit, V.; Elkin, J.; Denman, T.; Cooper, J. A.; Kotha, $S$. P. Large-scale and highly efficient synthesis of micro-and nano-fibers with controlled fiber morphology by centrifugal jet spinning for tissue regeneration. Nanoscale 2013, 5, 2337-2345.

(25) Mellado, P.; Mcllwee, H. A.; Badrossamay, M. R.; Goss, J. A.; Mahadevan, L.; Parker, K. K. A simple model for nanofiber formation by rotary jet-spinning. Appl. Phys. Lett. 2011, 99, 203107.

(26) Zhang, X.; Lu, Y. Centrifugal Spinning: An Alternative Approach to Fabricate Nanofibers at High Speed and Low Cost. Polym. Rev. 2014, 54, 677-701.

(27) Hammami, M. A.; Krifa, M.; Harzallah, O. Centrifugal force spinning of PA6 nanofibers-processability and morphology of solution-spun fibers. J. Text. Inst. 2014, 105, 637-647.

(28) Mahalingam, S.; Edirisinghe, M. Forming of Polymer Nanofibers by a Pressurised Gyration Process. Macromol. Rapid Commun. 2013, 34, 1134-1139.

(29) Hong, X.; Edirisinghe, M.; Mahalingam, S. Beads, beaded-fibers and fibers: Tailoring the morphology of poly (caprolactone) using pressurised gyration. Mater. Sci. Eng., C 2016, 69, 1373-1382.

(30) Mahalingam, S.; Ren, G. G.; Edirisinghe, M. J. Rheology and pressurised gyration of starch and starch-loaded poly (ethylene oxide). Carbohydr. Polym. 2014, 114, 279-287.

(31) Mahalingam, S.; Raimi-Abraham, B. T.; Craig, D. Q. M.; Edirisinghe, M. Formation of Protein and Protein-Gold Nanoparticle 
Stabilized Microbubbles by Pressurized Gyration. Langmuir 2015, 31, 659-666.

(32) Wu, X.; Mahalingam, S.; VanOosten, S. K.; Wisdom, C.; Tamerler, C.; Edirisinghe, M. New Generation of Tunable Bioactive Shape Memory Mats Integrated with Genetically Engineered Proteins. Macromol. Biosci. 2017, 17, 1600270.

(33) Zhang, S.; Karaca, B. T.; VanOosten, S. K.; Yuca, E.; Mahalingam, S.; Edirisinghe, M.; Tamerler, C. Coupling Infusion and Gyration for the Nanoscale Assembly of Functional Polymer Nanofibers Integrated with Genetically Engineered Proteins. Macromol. Rapid Commun. 2015, 36, 1322-1328.

(34) Hong, X.; Mahalingam, S.; Edirisinghe, M. Simultaneous Application of Pressure-Infusion-Gyration to Generate Polymeric Nanofibers. Macromol. Mater. Eng. 2017, 302, 1600564.

(35) Ketabchi, N.; Naghibzadeh, M.; Adabi, M.; Esnaashari, S. S.; Faridi-Majidi, R. Preparation and optimization of chitosan/polyethylene oxide nanofiber diameter using artificial neural networks. Neural Comput. Appl. 2017, 28, 3131-3143.

(36) Nasouri, K.; Shoushtari, A. M.; Khamforoush, M. Comparison between Artificial Neural Network and Response Surface Methodology in the Prediction of the Production Rate of Polyacrylonitrile Electrospun Nanofibers. Fibers Polym. 2013, 14, 1849-1856.

(37) Reisi-Dehkordi, A.; Eslami-Farsani, R. Prediction of High Performance Fibers Strength Using Back Propagation Neural Network. J. Macromol. Sci., Part A: Pure Appl.Chem. 2015, 52, 642647.

(38) Basheer, I. A.; Hajmeer, M. Artificial neural networks: fundamentals, computing, design, and application. J. Microbiol. Methods 2000, 43, 3-31.

(39) Carley, K. M.; Kamneva, N. Y.; Reminga, J. Response Surface Methodology; Defense Technical Information Center, 2004; ADA459032.

(40) Bezerra, M. A.; Santelli, R. E.; Oliveira, E. P.; Villar, L. S.; Escaleira, L. A. Response surface methodology (RSM) as a tool for optimization in analytical chemistry. Talanta 2008, 76, 965-977.

(41) Zheng, Y.; Liu, Y.; Wang, A. Fast removal of ammonium ion using a hydrogel optimized with response surface methodology. Chem. Eng. J. 2011, 171, 1201-1208.

(42) Acherjee, B.; Misra, D.; Bose, D.; Venkadeshwaran, K. Prediction of weld strength and seam width for laser transmission welding of thermoplastic using response surface methodology. Opt. Laser Technol. 2009, 41, 956-967.

(43) Wächter, R; Cordery, A. Response surface methodology modelling of diamond-like carbon film deposition. Carbon 1999, 37, 1529-1537. 